\title{
The covariance of cosmic shear correlation functions and cosmological parameter estimates using redshift information
}

\author{
P. Simon, L. J. King, and P. Schneider
}

Institut für Astrophysik und Extraterrestrische Forschung, Universität Bonn, Auf dem Hügel 71, 53121 Bonn, Germany

Received 1 September 2003 / Accepted 13 January 2004

\begin{abstract}
Cosmological weak lensing by the large scale structure of the Universe, cosmic shear, is coming of age as a powerful probe of the parameters describing the cosmological model and matter power spectrum. It complements Cosmic Microwave Background studies, by breaking degeneracies and providing a cross-check. Furthermore, upcoming cosmic shear surveys with photometric redshift information will enable the evolution of dark matter to be studied, and even a crude separation of sources into redshift bins leads to improved constraints on parameters. An important measure of the cosmic shear signal are the shear correlation functions; these can be directly calculated from data, and compared with theoretical expectations for different cosmological models and matter power spectra. We present a Monte Carlo method to quickly simulate mock cosmic shear surveys. One application of this method is in the determination of the full covariance matrix for the correlation functions; this includes redshift binning and is applicable to arbitrary survey geometries. Terms arising from shot noise and cosmic variance (dominant on small and large scales respectively) are accounted for naturally. As an illustration of the use of such covariance matrices, we consider to what degree confidence regions on parameters are tightened when redshift binning is employed. The parameters considered are those commonly discussed in cosmic shear analyses - the matter density parameter $\Omega_{\mathrm{m}}$, dark energy density parameter (classical cosmological constant) $\Omega_{\Lambda}$, power spectrum normalisation $\sigma_{8}$ and shape parameter $\Gamma$. We incorporate our covariance matrices into a likelihood treatment, and also use the Fisher formalism to explore a larger region of parameter space. Parameter uncertainties can be decreased by a factor of $\sim 4-8(\sim 5-10)$ with 2 (4) redshift bins.
\end{abstract}

Key words. cosmology: large-scale structure of Universe - cosmology: theory - gravitational lensing - methods: numerical

\section{Introduction}

The statistics of the distorted images of distant galaxies, gravitationally lensed by the tidal gravitational field of intervening matter inhomogeneities, contain a wealth of information about the power spectrum of the dark and luminous matter in the Universe, and the underlying cosmological parameters. The importance of "cosmic shear" as a cosmological tool was proposed in the early 1990s by Blandford et al. (1991), Miralda-Escudé (1991) and Kaiser (1992). Further analytic and numerical work (e.g. Kaiser 1998; Schneider et al. 1998; White \& Hu 2000) took into account the increased power on small scales, resulting from the non-linear evolution of the power spectrum (Hamilton et al. 1991; Peacock \& Dodds 1996).

The feasibility of cosmic shear studies was demonstrated in 2000, when four teams announced the first observational detections (Bacon et al. 2000; Kaiser et al. 2000; van Waerbeke et al. 2000; Wittman et al. 2000). Upcoming surveys will cover much larger areas, and multicolour observations will enable photometric redshift estimates for the galaxies to be obtained. For example, the CFHT Legacy survey (http://www. cfht. Hawaii.edu/Science/CFHLS) will

Send offprint requests to: P. Simon, e-mail: psimon@astro.uni-bonn.de cover $172 \operatorname{deg}^{2}$ in 5 optical bands, with a smaller area to be observed in $J$ and $K$ bands.

In order to compare these observations with predictions for various cosmological models and matter power spectra, different two-point statistics of galaxy ellipticities can be employed, all of which are filtered versions of the convergence power spectrum. Here, we focus on the gravitational shear correlation functions, which can be directly obtained from the data as described in Sect. 2.2.

This quest for the parameters describing the matter content and geometry of the Universe is limited by several sources of error, dominated by the dispersion in the intrinsic ellipticities of galaxies and by cosmic (sampling) variance. The covariance (error) matrix is thus an essential ingredient in the extraction of parameters from data, or in parameter error estimate predictions. Schneider et al. (2002a) provide analytical approximations for the contributions to the covariance matrix. They consider the case when the mean redshift of the population is known, and data taken in a single contiguous area. Kilbinger \& Schneider (2004) use a numerical approach to investigate the impact of survey geometry on parameter constraint. Using a Fisher matrix approach, which provides a lower-bound estimate of covariance, Hu (1999) has shown that even crude redshift information enables much tighter constraints to be placed 
on cosmological parameters, compared with the case when only the mean redshift of the population is known. This study concentrated on the convergence power spectrum as the vehicle of cosmological information.

Motivated by these studies, in this paper we demonstrate how numerical simulations can be used to estimate the full covariance matrix for the shear correlation functions in the presence of redshift information, and for arbitrary survey geometries. We consider auto- and cross-correlations for redshift bins (as in $\mathrm{Hu}$ 1999) and in addition allow for cross-correlations between measurements of the shear signal at different angular scales. With covariance matrices in hand, we then investigate the improvement in parameter estimates due to redshift binning.

Further details and derivations of the equations relevant to cosmic shear and weak lensing can be found in Bartelmann \& Schneider (2001). For a recent review of cosmic shear and future prospects see van Waerbeke \& Mellier (2003).

\section{Power spectrum and correlation functions}

Access to cosmological parameters is provided through the $o b$ servable two-point statistics of the ellipticities of distant galaxies. In this section, we describe how these are related to the matter power spectrum, and to the underlying density field.

\subsection{The convergence power spectrum}

The power spectrum $P_{K}(\ell)$ of the effective convergence, or equivalently of the shear $P_{\gamma}(\ell)$ (e.g. Bartelmann \& Schneider 2001), is related to that of the density fluctuations, $P_{\delta}(\ell)$, through a variant of Limber's equation in Fourier space (Kaiser 1998)

$$
\begin{aligned}
& P_{K}(\ell)=\frac{9 H_{0}^{4} \Omega_{\mathrm{m}}^{2}}{4 c^{4}} \int_{0}^{w_{\mathrm{H}}} \mathrm{d} w \frac{\bar{W}^{2}(w)}{a^{2}(w)} P_{\delta}\left(\frac{\ell}{f(w)}, w\right) \\
& \bar{W}(w) \equiv \int_{w}^{w_{\mathrm{H}}} \mathrm{d} w^{\prime} p\left(w^{\prime}\right) \frac{f\left(w^{\prime}-w\right)}{f\left(w^{\prime}\right)} \\
& f(w)= \begin{cases}K^{-1 / 2} \sin \left(K^{1 / 2} w\right) & (K>0) \\
w & (K=0), \\
(-K)^{-1 / 2} \sinh \left[(-K)^{1 / 2} w\right] & (K<0)\end{cases}
\end{aligned}
$$

where $\ell$ is the angular wave-vector, Fourier space conjugate to $\boldsymbol{\theta} . w$ is the comoving radial distance, $K$ the curvature parameter. A value $K=0$ corresponds to $\Omega_{\mathrm{m}}+\Omega_{\Lambda}=1$, where $\Omega_{\Lambda}$ is the cosmological constant and $\Omega_{\mathrm{m}}$ the matter density parameter. The function $\bar{W}(w)$ accounts for the sources being distributed in redshift, where $p\left(w^{\prime}\right) \mathrm{d} w^{\prime}$ is the comoving distance probability distribution for the sources. $a(w)$ is the scale factor normalised to $a(w=0)=1$ and $H_{0}$ is the Hubble constant.

Splitting up the weak lensing survey in redshift, as in Fig. 1, defines a set of effective convergence and shear maps instead of a single one, giving more information on the evolution of the dark matter fluctuations and therefore enabling tighter constraints to be placed on cosmological parameters. Auto- and

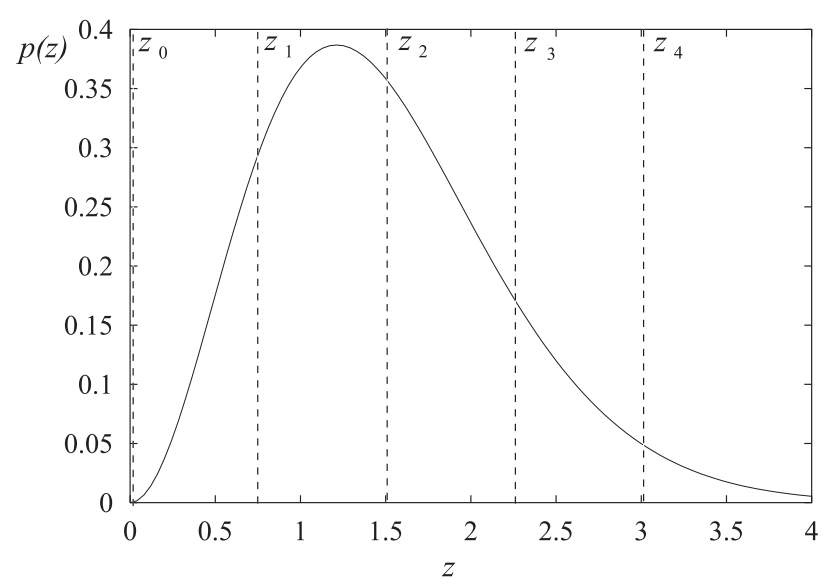

Fig. 1. Galaxies are binned together according to their redshift, the boundaries of the pairwise adjacent redshift bins are $w_{i}$ with $i=0 \ldots N_{z}$ (here as an example $N_{z}=4$ ). For every redshift bin the reduced shear field is calculated, averaging over the redshift distribution inside the bin.

cross-correlation of these maps introduce a whole set of power spectra, generalising Eq. (1):

$$
\begin{aligned}
P_{K}^{(i j)}(\ell)= & \frac{9 H_{0}^{4} \Omega_{\mathrm{m}}^{2}}{4 c^{4}} \\
& \times \int_{0}^{w_{H}} \mathrm{~d} w \frac{\bar{W}^{(i)}(w) \bar{W}^{(j)}(w)}{a^{2}(w)} P_{\delta}\left(\frac{\ell}{f(w)}, w\right) \\
\bar{W}^{(i)}(w) \equiv & \int_{w_{i-1}}^{w_{i}} \mathrm{~d} w^{\prime} p^{(i)}\left(w^{\prime}\right) \frac{f\left(w-w^{\prime}\right)}{f\left(w^{\prime}\right)}
\end{aligned}
$$

with $p^{(i)}(w)$ being the normalised distribution in comoving distance inside the $i$ th bin, where $i$ runs between 1 and the number of redshift bins $N_{z} . P_{\kappa}^{(i i)}$ are auto-correlation power spectra, whereas $P_{k}^{(i j)}$ with $i \neq j$ are cross-correlation power spectra.

\subsection{Shear correlation functions}

Constraints can be placed on cosmological parameters using the directly observable shear correlation functions, which we now turn to.

The basis which underpins the use of the distorted images of distant galaxies in weak lensing studies is a transformation relating the source, $\epsilon^{(\mathrm{s})}$, and image, $\epsilon$, (complex) ellipticities to the tidal gravitational field of density inhomogeneities (for definitions see Bartelmann \& Schneider 2001). We focus on the non-critical regime where

$\epsilon=\frac{\epsilon^{(\mathrm{s})}+g}{1+g^{*} \epsilon^{(\mathrm{s})}} \approx \epsilon^{(\mathrm{s})}+\gamma$,

where $g \equiv \gamma /(1-\kappa)$ is the reduced shear.

Empirically the probability distribution function (pdf) of the galaxies' intrinsic ellipticities is a truncated Gaussian for both the real and imaginary parts of $\epsilon^{(\mathrm{s})}$ :

$p_{\epsilon^{(\mathrm{s})}}=\frac{\exp \left(-\left|\epsilon^{(\mathrm{s})}\right|^{2} / \sigma_{\epsilon^{(\mathrm{s})}}^{2}\right)}{\pi \sigma_{\epsilon^{(\mathrm{s})}}^{2}\left[1-\exp \left(-1 / \sigma_{\epsilon^{(\mathrm{s})}}^{2}\right)\right]}$,

where $\sigma_{\epsilon^{(\mathrm{s})}}$ is the intrinsic ellipticity dispersion. 
As in Schneider et al. (2002b), the shear correlation functions are defined as

$\xi_{ \pm}(\theta)=\left\langle\gamma_{\mathrm{t}} \gamma_{\mathrm{t}}\right\rangle \pm\left\langle\gamma_{\times} \gamma_{\times}\right\rangle=\int_{0}^{\infty} \frac{\mathrm{d} \ell \ell}{2 \pi} J_{0,4}(\ell \theta) P_{\kappa}(\ell)$,

where $J_{n}$ are $n$th order Bessel functions of the first kind; $\gamma_{\mathrm{t}}$ and $\gamma_{\times}$are the tangential and cross shear components respectively. From now on we focus on $\xi_{+}$, since this contains most of the cosmological information on the scales of interest.

\subsection{Choice of cosmology and matter power spectrum}

Unless otherwise stated, our cosmology throughout is a $\Lambda \mathrm{CDM}$ model with $\Omega_{\mathrm{m}}=0.3, \Omega_{\Lambda}=0.7$ and $H_{0}=70 \mathrm{~km} \mathrm{~s}^{-1} \mathrm{Mpc}^{-1}$. A scale-invariant $(n=1$, Harrison-Zel'dovich) spectrum of primordial fluctuations is assumed. Predicting the shear correlation functions requires a model for the redshift evolution of the 3-D power spectrum. We use the fitting formula of Bardeen et al. (1986; BBKS) for the transfer function, and the Peacock \& Dodds (1996) prescription for evolution in the nonlinear regime. The power spectrum normalisation is parameterised with $\sigma_{8}=0.9$, and $\Gamma=0.21$. Quantities calculated for this fiducial cosmology/power spectrum will be super-scripted with a " $t$ ".

\section{Simulating cosmic shear surveys}

In this section we describe the method we used to make Monte-Carlo simulations of cosmic shear surveys. The implementation makes the simulations as computationally inexpensive as possible, i.e. without invoking $N$-body simulations.

Calculating the lensing signal by ray tracing through $\mathrm{N}$-body simulations has become a common tool for making simulated weak lensing surveys (see e.g. Blandford et al. 1991; Wambsganss et al. 1998; Jain et al. 2000).

We take a different path here, because only the two-point statistics of weak lensing is considered. This allows us to reduce the computational effort by expressing the fields of the shear and convergence as random Gaussian fields having the same power spectrum as the corresponding fields from the $N$-body approach. In the weak lensing regime, ray-tracing is well described by the Born approximation which ignores the effects of lens-lens coupling and deviations of light rays from the fiducial path (see White \& Hu 2000). The task of calculating the required power spectra then becomes relatively straightforward, because these can be shown to be linear functions of the three-dimensional evolving dark matter power spectrum. The accuracy of the results depends on how accurately that three-dimensional power spectrum is known.

Since we consider the two-point cosmic shear statistics in an area of relatively small angular size, we can represent the cosmic shear fields by random Gaussian fields in a flat sky approximation.

The practical advantage of simulating a single random Gaussian field $\delta(\boldsymbol{r})$ - a homogeneous and isotropic random Gaussian to be exact - is the fact that from the Fourier coefficients

$c_{\boldsymbol{k}}=\frac{1}{V} \int_{V} \mathrm{~d} \boldsymbol{r} \delta(\boldsymbol{r}) \exp (\mathrm{i} \boldsymbol{k} \cdot \boldsymbol{r})$ of such a random field only a pair of coefficients is correlated

$\left\langle c_{k} c_{-k^{\prime}}\right\rangle=\frac{1}{V} \delta_{k k^{\prime}} P_{k}$,

where $\delta_{\mathrm{D}}$ is the Dirac delta function and $P_{\boldsymbol{k}}$ is the power spectrum of the random field. The volume $V$ of the Gaussian field is in our case simply the area on the sky covered by the field.

In our work, however, the situation is a bit more complicated than that: the ellipticities of galaxies belonging to different redshift bins are correlated as well as the ellipticities of galaxies at different angular positions. Therefore, when more than one redshift bin is considered, several Gaussian fields cosmic shear maps - with prescribed cross-correlations have to be simulated simultaneously. A way to do this in general on a regular grid for real Gaussian fields is shown in the following subsection. The subsection thereafter explains how we used this approach for simulating mock cosmic shear surveys.

\subsection{Realisations of correlated Gaussian fields}

According to condition (9) the pair $c_{k}$ and $c_{-k}$ is correlated. In this section we restrict ourselves to real Gaussian fields with $\delta(\boldsymbol{r})=\delta^{*}(\boldsymbol{r})$. This introduces an additional condition that follows from the definition (8) of the $c_{k}$ :

$c_{\boldsymbol{k}}=c_{-\boldsymbol{k}}^{*}$.

In particular, for real Gaussian fields we thereby have

$\left\langle c_{k} c_{k^{\prime}}^{*}\right\rangle=\frac{1}{V} P_{k} \delta_{k k^{\prime}}$,

$\delta_{\boldsymbol{k} \boldsymbol{k}^{\prime}}$ being the Kronecker symbol. The conditions (9) and (10) are easily accounted for if, say, only the $c_{k}$ for half of the spatial frequencies $\boldsymbol{k}$ are worked out and the $c_{-\boldsymbol{k}}$ frequencies are set accordingly. Hence, for our choice, if we talk about $c_{k}$ we actually mean only Fourier coefficients in the right half-plane.

Furthermore, the real and imaginary parts of $c_{k}$ are uncorrelated, and both follow the same Gaussian pdf. This pdf has zero mean ${ }^{1}$ and a variance $\sigma_{k}$ that is expressed in terms of the power spectrum $P_{\boldsymbol{k}}$ describing the two-point correlations of the fluctuations in the Gaussian field (see e.g. Peacock 2001)

$\sigma_{k}^{2}=\frac{1}{2 V} P_{k}$

The procedure for making one Gaussian field realisation requires two steps: 1 . drawing numbers for the real and imaginary parts for every $c_{k}$ with a Gaussian random number generator, and 2. transformation of this Fourier space representation to real space in order to obtain the field realisation. For the second step we used an FFT algorithm from Press et al. $(1992)^{2}$.

This procedure also holds when realisations of more than one, but uncorrelated Gaussian fields are desired.

\footnotetext{
1 In the case that $\delta(\boldsymbol{r})$ has a non zero mean, $\left\langle c_{\boldsymbol{k}}\right\rangle$ for $\boldsymbol{k}=0$ becomes different from zero.

2 As in FFT the matrix of the Fourier coefficients contains $c_{k}$ that share the same matrix elements with $c_{-k}$, one has for these particular coefficients to set the imaginary parts to zero and to increase $\sigma_{k}$ by the factor $\sqrt{2}$. The latter is necessary to guarantee that the variance of the modulus of $c_{\boldsymbol{k}}$ is still correct.
} 
"Uncorrelated" means that if we denote the Fourier coefficients of, say, $N$ Gaussian random fields by $c_{\boldsymbol{k}}^{(i)}$ with $i=1 \ldots N$ then we expect for those fields the relation

$\left\langle c_{\boldsymbol{k}}^{(i)}\left[c_{\boldsymbol{k}^{\prime}}^{(j)}\right]^{*}\right\rangle=\frac{1}{V} P_{\boldsymbol{k}}^{(i i)} \delta_{i j} \delta_{\boldsymbol{k} \boldsymbol{k}^{\prime}}$,

where $\delta_{i j}$ is also a Kronecker symbol, this time for the Gaussian field indices. $P_{\boldsymbol{k}}^{(i i)}$ is the previously introduced power spectrum, or auto-correlation power spectrum, of the $i$ th random field. Thus, here correlations between $c_{\boldsymbol{k}}$ of different random fields vanish.

For the purposes of this work, however, we need to be able to allow for cross-correlations between different random fields $i \neq j$ in a defined manner, like

$\left\langle c_{\boldsymbol{k}}^{(i)}\left[c_{\boldsymbol{k}^{\prime}}^{(j)}\right]^{*}\right\rangle=\frac{1}{V} P_{\boldsymbol{k}}^{(i j)} \delta_{\boldsymbol{k} \boldsymbol{k}^{\prime}}$.

$P_{\boldsymbol{k}}^{(i j)}$ is for $i \neq j$ the cross-correlation power spectrum. Like for the auto-correlations, only certain pairs of Fourier coefficients of different Gaussian fields are correlated. This follows from the assumption that the cross-correlations are homogeneous, too. Note that $P_{\boldsymbol{k}}^{(i j)}=P_{\boldsymbol{k}}^{(j i)}$.

In order to find a recipe for making realisations of that kind, we make the Ansatz that the $N$ Fourier coefficients $c_{\boldsymbol{k}}^{(i)}$ are a linear transformation $\boldsymbol{A}_{\boldsymbol{k}}$ of $N$ different uncorrelated coefficients $d_{\boldsymbol{k}}^{(i)}$ with an equal Gaussian pdf for the real and imaginary parts, zero mean and a $1 / \sqrt{2}$ dispersion

$$
\left\langle d_{\boldsymbol{k}}^{(i)}\left[d_{\boldsymbol{k}}^{(j)}\right]^{*}\right\rangle=\delta_{i j} ; \quad c_{\boldsymbol{k}}^{(i)}=\sum_{q}\left[\boldsymbol{A}_{\boldsymbol{k}}\right]_{q}^{i} d_{\boldsymbol{k}}^{(q)} .
$$

$\boldsymbol{A}_{\boldsymbol{k}}$ is a $N \times N$ linear transformation matrix. The linearity of this transformation accounts for the fact that the resulting set of coefficients $c_{\boldsymbol{k}}^{(i)}$ still obeys a Gaussian statistics, because linear combinations of Gaussian random variables are also Gaussian.

Since for real Gaussian fields real and imaginary parts of the Fourier coefficients are not correlated, and by our Ansatz neither are the real and imaginary parts of $d_{\boldsymbol{k}}^{(i)}$, only real numbers for the components $\left[\boldsymbol{A}_{\boldsymbol{k}}\right]_{q}^{i}$ are allowed; an additional imaginary part of $\boldsymbol{A}_{\boldsymbol{k}}$ would mix real and imaginary parts of $d_{k}^{(i)}$, thereby possibly introducing correlations between real and imaginary parts in $c_{\boldsymbol{k}}$. A matrix $\boldsymbol{A}_{\boldsymbol{k}}$ that is purely imaginary would be an alternative choice, though.

Equations (15) can now be combined to give

$$
\begin{aligned}
\left\langle c_{\boldsymbol{k}}^{(i)}\left[c_{\boldsymbol{k}}^{(j)}\right]^{*}\right\rangle & =\sum_{q, r}\left\langle\left[\boldsymbol{A}_{\boldsymbol{k}}\right]_{q}^{i} d_{\boldsymbol{k}}^{(q)}\left[\boldsymbol{A}_{\boldsymbol{k}}\right]_{r}^{j}\left[d_{\boldsymbol{k}}^{(r)}\right]^{*}\right\rangle \\
& =\sum_{q, r}\left[\boldsymbol{A}_{\boldsymbol{k}}\right]_{q}^{i}\left[\boldsymbol{A}_{\boldsymbol{k}}\right]_{r}^{j}\left\langle d_{\boldsymbol{k}}^{(q)}\left[d_{\boldsymbol{k}}^{(r)}\right]^{*}\right\rangle \\
& =\sum_{q, r}\left[\boldsymbol{A}_{\boldsymbol{k}}\right]_{q}^{i}\left[\boldsymbol{A}_{\boldsymbol{k}}\right]_{r}^{j} \delta_{q r} \\
& =\sum_{q}\left[\boldsymbol{A}_{\boldsymbol{k}}\right]_{q}^{i}\left[\boldsymbol{A}_{\boldsymbol{k}}^{T}\right]_{j}^{q},
\end{aligned}
$$

where $\boldsymbol{A}_{\boldsymbol{k}}^{T}$ denotes the transpose of $\boldsymbol{A}_{\boldsymbol{k}}$. Hence, together with Eq. (14) this puts another constraint on the matrix $\boldsymbol{A}_{\boldsymbol{k}}$, namely

$$
\frac{1}{V} P_{\boldsymbol{k}}^{(i j)}=\sum_{q}\left[\boldsymbol{A}_{\boldsymbol{k}}\right]_{q}^{i}\left[\boldsymbol{A}_{\boldsymbol{k}}^{T}\right]_{j}^{q} \text {. }
$$

For convenience we introduce the power matrix defined as $\left[\boldsymbol{P}_{\boldsymbol{k}}\right]_{j}^{i} \equiv \frac{1}{V} P_{\boldsymbol{k}}^{(i j)}$ to abbreviate this equation:

$$
\boldsymbol{P}_{k}=\boldsymbol{A}_{k} \boldsymbol{A}_{k}^{T} \text {. }
$$

The power matrix is the covariance matrix between the Fourier coefficients of a set of Gaussian fields for a certain $\boldsymbol{k}$.

This shorthand of $N^{2}$ equations does not uniquely determine the matrix $\boldsymbol{A}_{\boldsymbol{k}}$, because it contains only $N(N+1) / 2$ linearly independent equations, since both the matrix on the lhs and the matrix product on the rhs are symmetric. As there are no further constraints on $\boldsymbol{A}_{\boldsymbol{k}}$, we are allowed to set the remaining $N^{2}-N(N+1) / 2=N(N-1) / 2$ constraints of $\boldsymbol{A}_{\boldsymbol{k}}$ as we like. We do this by assuming that $\boldsymbol{A}_{\boldsymbol{k}}$ is symmetric, so that we finally obtain

$$
\boldsymbol{P}_{k}=A_{k}^{2} \Rightarrow A_{k}=\sqrt{\boldsymbol{P}_{k}}
$$

In general the square root is not unique (see e.g. Higham 1997). However, we are already satisfied with one particular solution to this problem. In order to determine such a solution, note that $\boldsymbol{P}_{\boldsymbol{k}}$ is a symmetric positive (semi)definite matrix, which is ensured by the properties of the power spectra the power matrix consists of:

$$
\begin{gathered}
P_{\boldsymbol{k}}^{(i j)}=P_{\boldsymbol{k}}^{(j i)}, \\
{\left[P_{\boldsymbol{k}}^{(i j)}\right]^{2} \leq P_{\boldsymbol{k}}^{(i i)} P_{\boldsymbol{k}}^{(j j)} .}
\end{gathered}
$$

Therefore, this matrix can uniquely be decomposed into

$\boldsymbol{P}_{\boldsymbol{k}}=\boldsymbol{R}_{\boldsymbol{k}}^{T} \boldsymbol{D}_{\boldsymbol{k}} \boldsymbol{R}_{k}$

where $\boldsymbol{R}_{\boldsymbol{k}}$ is an orthogonal matrix whose column vectors are the eigenvectors of $\boldsymbol{P}_{\boldsymbol{k}}$, while their corresponding, always real and positive, eigenvalues $\lambda_{i}$ are on the diagonal of the diagonal matrix $\boldsymbol{D}_{\boldsymbol{k}}=\operatorname{diag}\left(\lambda_{1}, \lambda_{2}, \ldots, \lambda_{\mathrm{N}}\right)$. As one particular square root we pick out

$$
\begin{aligned}
\boldsymbol{A}_{\boldsymbol{k}} & =\boldsymbol{R}_{\boldsymbol{k}}^{T} \sqrt{\boldsymbol{D}_{\boldsymbol{k}}} \boldsymbol{R}_{\boldsymbol{k}} \\
\sqrt{\boldsymbol{D}_{\boldsymbol{k}}} & \equiv \operatorname{diag}\left(\sqrt{\lambda_{1}}, \sqrt{\lambda_{2}}, \ldots, \sqrt{\lambda_{N}}\right)
\end{aligned}
$$

which is a solution due to $\sqrt{D_{k}} \sqrt{D_{k}}=D_{k}$.

To sum up, for every $\boldsymbol{k}$ mode considered, the process for the realisation of correlated Gaussian random fields requires one to find the square root $\boldsymbol{A}_{\boldsymbol{k}}$ of the power matrix $\boldsymbol{P}_{\boldsymbol{k}}$. This defines a linear transformation for a vector of uncorrelated random complex numbers (real and imaginary part of the same coefficient are uncorrelated, too) with zero mean, real and imaginary parts obeying a Gaussian pdf with $1 / \sqrt{2}$ variance. Applying $\boldsymbol{A}_{\boldsymbol{k}}$ yields a vector of Fourier coefficients belonging to the realisations of the correlated Gaussian random fields. Due to (10) this is performed only for one half of the spatial frequencies considered. The other half is set accordingly to fulfil this condition.

\subsection{Simulating the weak lensing survey}

Each galaxy in the mock galaxy catalogue is defined by an angular position, an ellipticity $\epsilon$ and a redshift bin it belongs to. 
The ellipticity of the isophotes of a galaxy is determined by the intrinsic shape of a galaxy $\epsilon_{i}^{(\mathrm{s})}$ and the reduced shear $g$ at the position of the galaxy (see Eq. (5)). The reduced shear is a function of the convergence $\kappa$ and shear $\gamma$ which have to be simulated for each redshift bin as a map covering the simulated area. Here we assume that the galaxies are binned into $N_{z}$ pairwise adjacent redshift bins, chopping off the redshift distribution

$p(z)=\frac{1}{z_{0}^{3}} \frac{1}{\Gamma(3 / \beta) \beta} z^{2} \exp \left[-\left(\frac{z}{z_{0}}\right)^{\beta}\right]$

as in Fig. 1. This empirical distribution with $\beta=1.5$ and $z_{0}=1.0$ is based on deep field surveys (see e.g. Smail et al. 1995). The total number of galaxies inside the field, with chosen size of $5^{\circ} \times 5^{\circ}$, is set to be $\approx 2.7 \times 10^{6}$, to get an average of 30 galaxies per $\operatorname{arcmin}^{2}$. Moreover, the galaxies are assumed to be randomly distributed over the field of view.

The method of the last subsection is used to work out the convergence maps in Fourier space on a grid of $2048 \times 2048$ pixels for all redshift bins. As input the power matrix $\boldsymbol{P}_{\boldsymbol{k}}$, consisting of the auto- and cross-correlation power spectra of these convergence maps, specified by the Eqs. (4), is needed.

In the next step, the shear maps are obtained from the convergence maps using the relation

$\tilde{\gamma}_{\ell}=\frac{\ell_{1}^{2}-\ell_{2}^{2}+2 \mathrm{i} \ell_{1} \ell_{2}}{\ell_{1}^{2}+\ell_{2}^{2}} \tilde{\kappa}_{\ell} \quad \boldsymbol{\ell} \equiv\left(\begin{array}{l}\ell_{1} \\ \ell_{2}\end{array}\right)$.

$\tilde{\gamma}_{\ell}$ and $\tilde{\kappa}_{\ell}$ are the Fourier coefficients of the shear and convergence fields for the angular frequency $\boldsymbol{\ell}$, respectively. This relation stems from the fact that both shear and convergence are linearly related to a potential function. For every galaxy, shear and convergence are then combined with the intrinsic ellipticity $\epsilon_{i}^{(\mathrm{s})}$, randomly drawn from the pdf Eq. (6) using $\sigma_{\epsilon}^{(\mathrm{s})}=0.3$, to compute the final ellipticity of the galaxy via Eq. (5).

Both angular size $\Delta$ and number of pixels $N_{P}$ along one axis - the sampling size - limits the number of fluctuation modes accounted for in the simulated data. This means, since we are lacking fluctuations on scales outside of $5^{\circ} /\left(2 N_{P}\right) \leq$ $\Theta \leq 5^{\circ}$, equivalent to $\ell_{\min } \leq \ell \leq \ell_{\max }$, that we have less correlation in the cosmic shear fields than expected (Eq. (7))

$$
\begin{aligned}
\xi_{ \pm}(\theta) & =\int_{\ell_{\min }}^{\ell_{\max }} \frac{\mathrm{d} \ell \ell}{2 \pi} \mathrm{J}_{0,4}(\ell \theta) P_{\kappa}(\ell) \\
\ell_{\min } & =\frac{\pi}{\sqrt{2} \Delta} ; \quad \ell_{\max }=N_{P} \ell_{\min } .
\end{aligned}
$$

The values for the limits are estimates for a square field; the limits are not clearly defined, because the number of $\ell$-modes in the FFT matrix becomes very small near the cutoffs. One solution to this problem is to artificially set a clearly defined range within the interval $\left[\ell_{\min }, \ell_{\max }\right]$, or, as we have done, to find a best fit cutoff. This is found by varying the cutoffs to obtain closest agreement between the theoretical two-point correlation and the ensemble average of all Monte-Carlo realisations.

In total, we simulated two data sets. The first data set consists of $N_{f}=795$ independent realisations each $5^{\circ} \times 5^{\circ}$. The
Table 1. The final column denotes the name given to a particular binning of data. The entries in the columns $z_{i}$ show the corresponding cuts in redshift, $z_{\mathrm{cut}}$.

\begin{tabular}{llllll}
\hline \hline$z_{0}$ & $z_{1}$ & $z_{2}$ & $z_{3}$ & $z_{4}$ & Name \\
\hline 0 & 0.75 & 1.5 & 2.25 & 3.0 & 4 bins \\
0 & 1.5 & 2.25 & 3.0 & & 3 binsI \\
0 & 0.75 & 2.25 & 3.0 & 3 binsII \\
0 & 0.75 & 1.5 & 3.0 & 3 binsIII \\
0 & 2.25 & 3.0 & & 2 binsI \\
0 & 1.5 & 3.0 & & 2 binsII \\
0 & 0.75 & 3.0 & & 2 binsIII \\
0 & 1.25 & 3.0 & & & 2binsIV \\
0 & 3.0 & & & & onebin \\
\hline
\end{tabular}

redshift distribution of the galaxies was split into 2 bins at a redshift cut $z_{\text {cut }}=1.25$, and the distribution is truncated at $z=3$. For our fiducial surveys, we randomly selected 10 sub-fields, each of $1.25^{\circ} \times 1.25^{\circ}$, from different large realisations. This was done for two reasons: 1 . to reduce the computation time, since for 10 shear maps we require only one realisation, and 2 . sub-fields are less affected by $\ell_{\min }$ that necessarily enters the simulations due to the finite realisation area.

The second data set has 4 redshift bins, with $z_{\text {cut }}=$ $0.75,1.5,2.25,3.0$ where the last value is the truncation redshift. It has $N_{f}=266$ independent realisations. The fiducial surveys from this data set consist of single sub-fields of size $1.25^{\circ} \times 1.25^{\circ}$.

For both data sets, $\xi_{+}$was estimated (see next section) for $N_{\Delta \theta}=65$ angular separation bins, ranging from about $2^{\prime} .0$ to 40.0. For the first (second) data set, the correlation functions were subsequently averaged for 10 (1) sub-fields in order to simulate cosmic shear surveys consisting of 10 (1) independent data fields.

In a further step, the cross- and auto-correlation of the cosmic shear between the shear maps were, according to Appendix A, combined to yield the cosmic shear correlations for a coarser redshift binning; in each step the number of redshift bins was reduced by one by combining two neighbouring bins. This process gave for the first data set, apart from the original data, the shear correlation of one redshift bin with boundaries $z=0$ and $z=3$. The second data set allows more freedom of choice for combining redshift bins, so that we are able to construct several data sets with three and two redshift bins. Table 1 lists the different redshift bins and reference names, all extracted from the two original data sets.

Below, this data is used to study the improvement in the statistical uncertainties of the cosmological parameter estimates, if one has more information on the redshifts of the galaxies. 


\section{Estimating $\xi_{+}$}

To estimate the two-point correlator $\xi_{+}$between the galaxy ellipticities $\epsilon_{i}$-depending on position $\boldsymbol{\Theta}_{i}$ and redshift bin - inside of the sub-fields we use the estimator

$$
\begin{aligned}
\hat{\xi}_{+}(\theta)= & \frac{1}{N_{p}(\theta)} \\
& \times \sum_{i j} w_{i} w_{j}\left(\epsilon_{i \mathrm{t}} \epsilon_{j \mathrm{t}}+\epsilon_{i \times} \epsilon_{j \times}\right) \Delta_{\theta}\left(\left|\boldsymbol{\Theta}_{i}-\boldsymbol{\Theta}_{j}\right|\right) \\
N_{p}(\theta)= & \sum_{i j} w_{i} w_{j} \Delta_{\theta}\left(\left|\boldsymbol{\Theta}_{i}-\boldsymbol{\Theta}_{j}\right|\right) \\
\Delta_{\theta}(\phi) \equiv & \begin{cases}1 & \text { for } \theta-\Delta \theta / 2<\phi \leq \theta+\Delta \theta / 2 \\
0 & \text { otherwise }\end{cases}
\end{aligned}
$$

as mentioned in Schneider et al. (2002a), with $\Delta \theta$ being the width of the angular bins. Since we are dealing with simulated data here, there is no need to weight galaxies with respect to their ellipticity. Therefore, we set $w_{i}=1$ for every galaxy.

Although mathematically simple, it takes quite a time to evaluate the estimator due to the large number of galaxy pairs. To speed up the whole procedure we put a grid of rectangular cells of size $\Delta \theta \times \Delta \theta$ over the sub-field in question and compute the number $N_{i j}$ of galaxies and the mean of their ellipticities $\bar{\epsilon}_{i j}$ inside every cell. The index $i j$ indicates the position of the cell inside the grid. This means we are representing galaxies inside the same cell by a single data point with weight $N_{i j}$ and ellipticity $\bar{\epsilon}_{i j}$. In particular, all galaxies inside this cell are assumed to be placed at the same position. The estimator of $\xi_{+}$for this rearranged data set can be shown to be

$$
\begin{aligned}
\hat{\xi}_{+}(\theta)= & \frac{1}{N_{p}(\theta)} \\
& \times \sum_{i j, k l} N_{i j} N_{k l}\left(\bar{\epsilon}_{i j t} \bar{\epsilon}_{k l \mathrm{t}}+\bar{\epsilon}_{i j \times} \bar{\epsilon}_{k l \times}\right) \Delta_{\theta}\left(\left|\boldsymbol{\Theta}_{i j}-\boldsymbol{\Theta}_{k l}\right|\right) \\
N_{p}(\theta)= & \sum_{i j, k l} N_{i j} N_{k l} \Delta_{\theta}\left(\left|\boldsymbol{\Theta}_{i j}-\boldsymbol{\Theta}_{k l}\right|\right)
\end{aligned}
$$

where $\boldsymbol{\Theta}_{i j}$ represents the angular position of cell $i j$.

The advantage of this approach is obvious: instead of considering $N^{2}$ pairs ( $N$ is the number of galaxies) we have to consider only $N_{\mathrm{c}}^{2}$ pairs, where $N_{\mathrm{c}}$ is the number of grid cells. Thus, the number of pairs depends only on the cell size and not on the number of galaxies. Hence, this method pays off once the cell size becomes large enough, making the number of cells smaller than the number of galaxies. Moreover, in order to find all galaxies at some distance from a certain cell we no longer have to check all galaxies, but only neighbouring cells which are easy to find by the grid index.

The approach becomes inaccurate, however, for small angular bins, because for these the assumption that cell-galaxies are essentially concentrated into one single point is particularly inaccurate. By comparing the ensemble average of $\hat{\xi}_{+}$with the theoretical $\xi_{+}$we find that after the third angular bin this approximation becomes accurate enough. For our purposes, this approach is completely sufficient. A better and more sophisticated approach can be found in Pen \& Zhang (2003).
For the case with $N_{z}=2$, with the division at $z_{\text {cut }}=1.25$ (2binsIV) Fig. 4 shows the close agreement between the correlation and cross-correlation functions, averaged over 7950 subfields, with the analytical prediction for the fiducial $\Lambda \mathrm{CDM}$ cosmological model, obtained from Eq. (7). Shown are comparisons for the lower (L) and upper (U) redshift bins, and cross-correlation (LU). To account for finite field size in our numerical work, $\ell_{\min }=2 \pi / 14.9^{\circ}$ in the integration. As noted above, since there is no well-defined cut-off, this value of $\ell_{\text {min }}$ was determined by allowing it to vary while performing a leastsquares fit of $\xi_{\mathrm{L}, \mathrm{U}, \mathrm{LU}}^{\mathrm{t}}$ to $\left\langle\hat{\xi}_{\mathrm{L}, \mathrm{U}, \mathrm{LU}}\right\rangle$, so obtaining the inverse variance weighted mean $\ell_{\text {min }}$. A cut-off at high $\ell$ is not critical since in this regime the power-spectrum amplitude is much lower.

\section{Estimating the covariance of $\hat{\xi}_{ \pm}$}

We now outline how the covariance matrix of $\hat{\xi}_{ \pm}$is estimated, for the case of $N_{z}=2$ redshift bins, with the division at $z_{\text {cut }}=1.25$ (2binsIV). As described above, our mock survey consists of 10 uncorrelated fields. An angle bracket denotes averaging over all 7950 sub-fields. Note that we may drop the L, $\mathrm{U}$ and LU sub-scripts for ease of notation.

When no redshift binning is assumed, it is computationally advantageous to determine the shear correlation function by combining those determined for the case with redshift binning: $\hat{\xi}=n_{\mathrm{L}}^{2} \hat{\xi}_{\mathrm{L}}+2 n_{\mathrm{L}} n_{\mathrm{U}} \hat{\xi}_{\mathrm{LU}}+n_{\mathrm{U}}^{2} \hat{\xi}_{\mathrm{U}}$

where $n_{\mathrm{L}, \mathrm{U}}$ are the fraction of sources in the lower and upper bins respectively. A more general relation between $\hat{\xi}$ and the cross- and auto-correlations of the shear from more than two redshift bins can be found in Appendix A.

The impact of cosmic variance is taken into account by determining correlation functions for each of the $N_{f}=795$ independent surveys. The covariance matrix between bin $i$ and $j$ is determined using

$\boldsymbol{C}_{i j}=\left\langle(\hat{\xi}-\langle\hat{\xi}\rangle)_{i}(\hat{\xi}-\langle\hat{\xi}\rangle)_{j}\right\rangle_{N_{f}}$

where the outer average is performed over the $N_{f}=795$ surveys. If redshift binning is considered, there are $N_{z}\left(N_{z}+1\right) / 2$ combinations of correlation and cross-correlation functions and hence $\boldsymbol{C}$ is a matrix composed of $\left[N_{z}\left(N_{z}+1\right) / 2\right]^{2}$ blocks. Figure 2 illustrates this for the simplified case where $N_{z}=2$ and $N_{\Delta \theta}=5$; for example the block in the upper left of the matrix corresponds to elements $\boldsymbol{C}_{i j}=\left\langle\left(\hat{\xi}_{\mathrm{L}}-\langle\hat{\xi}\rangle_{\mathrm{L}}\right)_{i}\left(\hat{\xi}_{\mathrm{L}}-\langle\hat{\xi}\rangle_{\mathrm{L}}\right)_{j}\right\rangle$, and the shaded entry to $\boldsymbol{C}_{2,3}$. The block in the middle row, left column, corresponds to covariance elements between the crosscorrelation and the lower redshift bin, with the shaded entry being $\boldsymbol{C}_{8,2}$. The bins denoted by $i$ and $j$ extend over $\Delta \theta$ bins, repeated for each redshift auto- and cross-correlation bin.

A representation of the covariance matrix determined from our simulations with $N_{z}=4$ is shown in Fig. 3. Note that $\boldsymbol{C}$ has a strong diagonal, although it is not strictly diagonally dominant.

Our covariance matrix for the case of no redshift binning is consistent $(<10 \%$ difference) with the treatment of Schneider et al. (2002a), and with Kilbinger \& Schneider (2004) who adopted the same assumption of Gaussianity. 


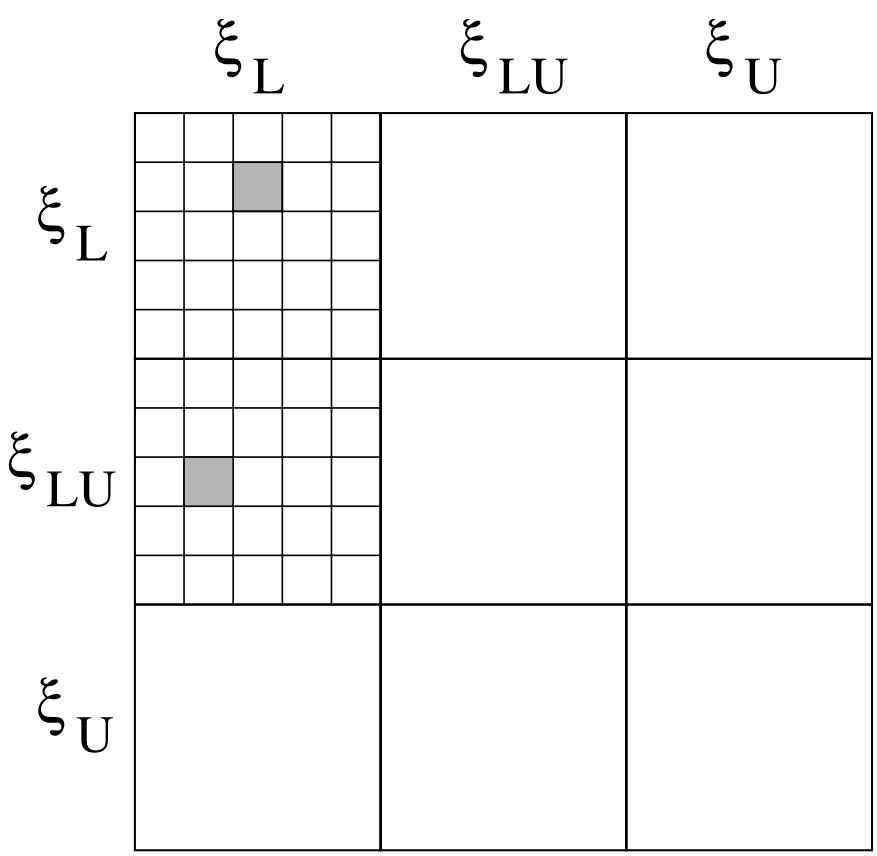

Fig. 2. Schematic illustration of the symmetric covariance matrix $\boldsymbol{C}$ for the case where there are $N_{z}=2$ source redshift bins and $N_{\Delta \theta}=5$ angular separation bins. Combinations of $\xi_{\mathrm{L}, \mathrm{U}, \mathrm{LU}}$ identify covariance terms of the form given in Eq. (31).

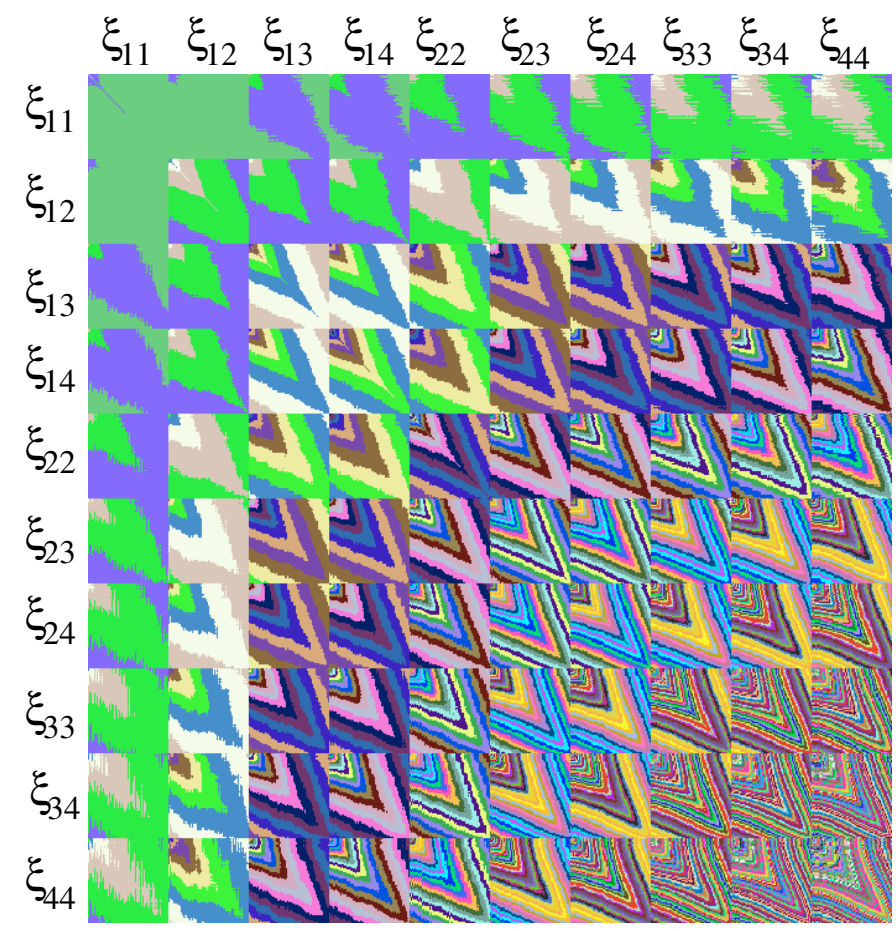

Fig. 3. The covariance matrix $\boldsymbol{C}$ determined from our simulations, for $N_{z}=4, N_{\Delta \theta}=65$ and a survey consisting of 1 sub-field. Different blocks correspond to auto- and cross-correlations between the redshift bins. Inside these blocks are auto-and cross-correlations for angular separation bins.

\section{An application: Constraints on cosmological parameters}

The rather featureless two-point shear correlation function $\xi_{+}(\theta)$ or corresponding convergence (shear) power

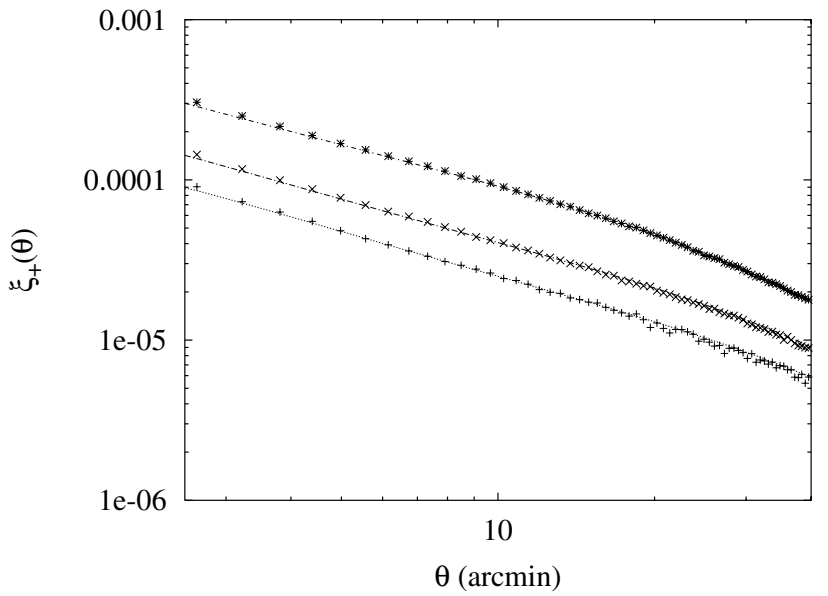

Fig. 4. Comparison of the analytical (lines) and numerical (symbols) shear correlation and cross-correlation functions $\xi_{\mathrm{L}, \mathrm{LU}, \mathrm{U}}$ (lower, middle and upper lines/symbols).

spectrum $P_{K}(\ell)$ leads to strong degeneracies amongst the parameters that can be derived from cosmic shear surveys. An indication of the degree of degeneracy is the behaviour of the partial derivatives of $\xi_{+}$with respect to each parameter $\pi_{i}$ (see King \& Schneider 2003 for such a comparison), or using a Fisher matrix analysis as in Sect. 6.2.1.

External sources of information often provide complimentary constraints: for example, confidence regions in the $\Omega_{\mathrm{m}}-\sigma_{8}$ plane derived from weak lensing are almost orthogonal to those from the analysis of CMB data (e.g. van Waerbeke et al. 2002), lifting this well known degeneracy (e.g. Bernardeau et al. 1997).

In this section we consider the extent to which crude redshift information for sources used in a lensing analysis decreases the expected errors in the $\Omega_{\mathrm{m}}-\sigma_{8}, \Omega_{\mathrm{m}}-\Gamma$ and $\sigma_{8}-\Gamma$ planes. Since we are interested in the influence of redshift binning on parameter degeneracies, hidden parameters are assumed to be perfectly known. As described above, we focus on the information provided by the shear two-point correlation function $\xi_{+}$(we may drop the " ")" subscript). We restrict this application to the case of $N_{z}=2$ (2binsIV). A larger parameter space is then explored using the covariance matrix derived from simulations in a Fisher analysis for the cases $N_{z}=2,3,4$.

\subsection{Obtaining confidence regions in the $\Omega_{m}-\sigma_{8}$, $\Omega_{m}-\Gamma$ and $\sigma_{8}-\Gamma$ planes}

We now determine and compare the likelihood contours in the $\Omega_{\mathrm{m}}-\sigma_{8}, \Omega_{\mathrm{m}}-\Gamma$ and $\sigma_{8}-\Gamma$ planes for the cases with and without redshift binning. The likelihood function is given by

$$
\begin{aligned}
\mathcal{L}(\pi)= & \frac{1}{(2 \pi)^{n / 2}|\boldsymbol{C}|^{1 / 2}} \\
& \times \prod_{i j} \exp \left[-\frac{1}{2}\left(\xi^{\mathrm{t}}-\xi(\pi)\right)_{i}\left[\boldsymbol{C}^{-1}\right]_{i j}\left(\xi^{\mathrm{t}}-\xi(\pi)\right)_{j}\right],
\end{aligned}
$$

where $n$ is the number of rows (or columns) of the covariance matrix $\boldsymbol{C}$ and $\xi(\pi)$ are theoretical correlation functions determined on a grid in parameter space. 


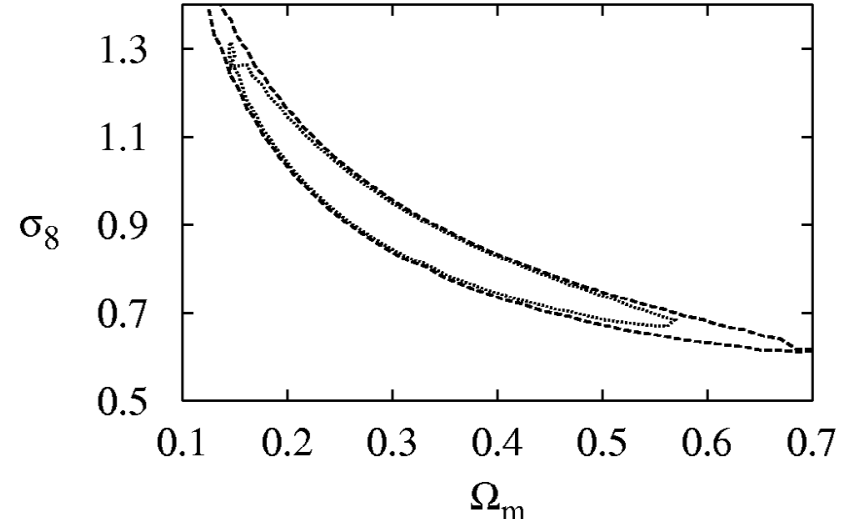

Fig. 5. Expected constraints in the $\Omega_{\mathrm{m}}-\sigma_{8}$ plane plotted for $\Delta \chi^{2}=4.61$ (90\% confidence) with (inner contour) and without (outer contour) redshift binning. The survey consists of 10 square uncorrelated sub-fields, each $1.25^{\circ}$ on a side. The redshift distribution and binning are described in the text.

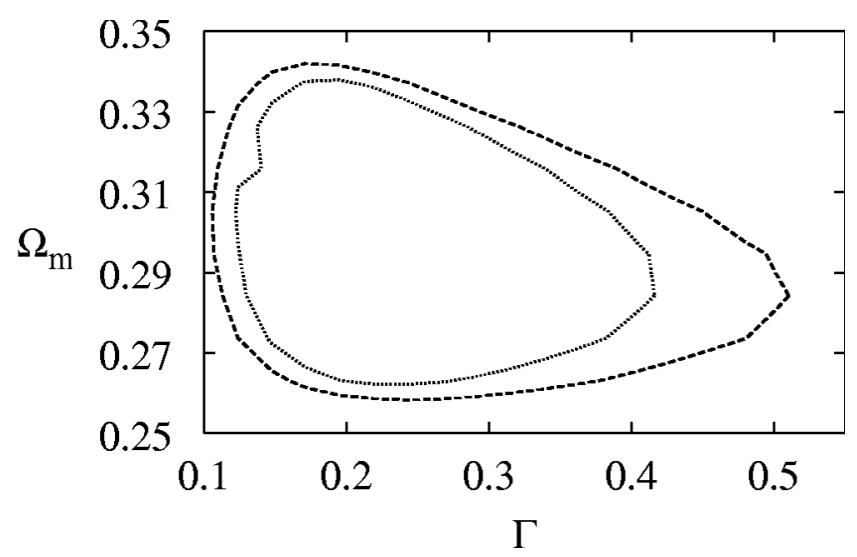

Fig. 6. Expected constraints in the $\Gamma-\Omega_{\mathrm{m}}$ plane plotted for $\Delta \chi^{2}=4.61$ (90\% confidence) with (inner contour) and without (outer contour) redshift binning. The survey is the same as in Fig. 5.

The log-likelihood function is distributed as $\chi^{2} / 2$ so that

$\chi^{2}(\pi)=\sum_{i j}\left(\xi^{\mathrm{t}}-\xi(\pi)\right)_{i}\left[\boldsymbol{C}^{-1}\right]_{i j}\left(\xi^{\mathrm{t}}-\xi(\pi)\right)_{j}$.

Confidence contours can be drawn in this $\chi^{2}$-surface, relative to the minimum (zero) at $\xi(\pi) \equiv \xi^{\mathrm{t}}$. In Figs. 5-7 the confidence contours are shown for each of the $\Omega_{\mathrm{m}}-\sigma_{8}, \Omega_{\mathrm{m}}-\Gamma$ and $\Gamma-\sigma_{8}$ planes, with and without redshift binning. Note that while $\Omega_{\mathrm{m}}$ is varied, we keep $\Omega_{\mathrm{m}}+\Omega_{\Lambda}=1$. To highlight the difference and avoid confusion, we plot contours for a single value of $\Delta \chi^{2}$.

\subsection{Fisher information}

The Fisher matrix (Fisher 1935) gives a handle on the question as to how accurately model parameters can be estimated from a given data set. In this section, we will use this method to examine quantitatively the increase of information on the cosmological parameters $\Omega_{\mathrm{m}}, \Omega_{\Lambda}, \sigma_{8}$ and $\Gamma$ when the number of redshift bins, thus the knowledge of the three-dimensional distribution of the galaxies, is increased. Note that we no longer impose the

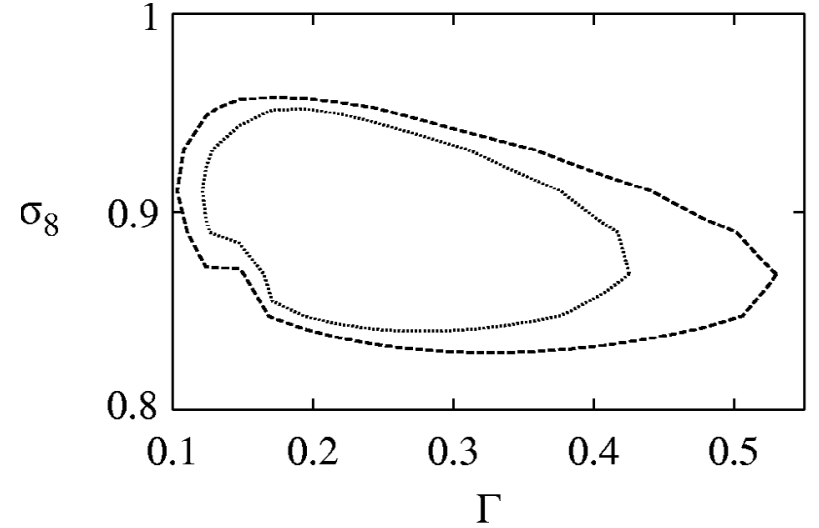

Fig. 7. Expected constraints in the $\Gamma-\sigma_{8}$ plane plotted for $\Delta \chi^{2}=4.61$ ( $90 \%$ confidence) with (inner contour) and without (outer contour) redshift binning. The survey is the same as in Fig. 5.

condition $\Omega_{\mathrm{m}}+\Omega_{\Lambda}=1$. After a brief introduction to this topic we apply the Fisher statistics to our simulated data.

\subsubsection{Fisher Formalism}

In general, one uses data points $\xi_{i}$ from a measurement to infer model parameters $\pi_{i}$ based on a theoretical model. As the measurements are polluted by noise, we cannot expect to exactly obtain the data points $\xi(\pi)$ predicted by our model. But we can try to find a combination of model parameters $\hat{\pi}_{i}$ that predict data points as close as possible to the actual measurement. The closeness is decided on the grounds of a statistical estimator. The covariance of the parameter uncertainties

$Q_{i j} \equiv\left\langle\Delta \pi_{i} \Delta \pi_{j}\right\rangle$

with $\Delta \pi_{i} \equiv\left(\left\langle\hat{\pi}_{i}^{2}\right\rangle-\left\langle\hat{\pi}_{i}\right\rangle^{2}\right)^{1 / 2}$ is related to the so-called Fisher information matrix through

$F_{i j} \equiv-\left\langle\frac{\partial^{2} \log \mathcal{L}[\pi, \xi]}{\partial \pi_{i} \partial \pi_{j}}\right\rangle=\left\langle\left[Q^{-1}\right]_{i j}\right\rangle$.

$\mathcal{L}$ corresponds to the likelihood for obtaining the measurement $\xi$ keeping the underlying model parameter $\pi_{i}$ fixed. See for example Tegmark et al. (1997) and references therein for a more detailed description.

It follows from statistics that the $1 \sigma$ scatter of the estimated parameters is (Cramér-Rao inequality)

$\Delta \pi_{i} \geq \sqrt{\left[\boldsymbol{F}^{-1}\right]_{i i}}$

where commonly the lower limit is taken to be the estimate for $\Delta \pi_{i}$. To quantify the degeneracies in the parameter estimate, we evaluate the correlation of the estimate's uncertainty contained in $\boldsymbol{F}$ :

$r_{i j} \equiv \frac{\left\langle\Delta \pi_{i} \Delta \pi_{j}\right\rangle}{\sqrt{\left\langle\Delta \pi_{i}^{2}\right\rangle\left\langle\Delta \pi_{j}^{2}\right\rangle}}=\frac{\left[\boldsymbol{F}^{-1}\right]_{i j}}{\sqrt{\left[\boldsymbol{F}^{-1}\right]_{i i}\left[\boldsymbol{F}^{-1}\right]_{j j}}}$

as, for example, in Huterer (2002). Highly correlated or anticorrelated $\Delta \pi_{i}$ and $\Delta \pi_{j}$ are called degenerate, whereas no correlation means no degeneracy (for the fiducial model). Another 
piece of information that can be extracted from the Fisher matrix is the orientation of the error ellipsoid in parameter space, which is defined by the eigenvectors of $\boldsymbol{F}$. This corresponds to the directions of degeneracies.

In the application of this formalism in Sect 6.2.2 below, we will look at situations where some of the model parameters are assumed to be known a priori. In this case, they are no longer free parameters that have to be estimated from measured data points, so that the size of the Fisher matrix reduces according to the number of parameters fixed. This amounts to removing rows and columns from the general Fisher matrix, one for each fixed parameter, so that these cases can be considered by simply looking at sub-matrices of the largest Fisher matrix. Taking all conceivable sub-matrices enables the exploration of all possible combinations of fixed (strong prior) and free parameters.

In practice, one uses the approximation (32) for the likelihood function $\mathcal{L}$ (where $\xi^{\mathrm{t}} \equiv \xi$ ), so that the Fisher information matrix is approximately

$F_{i j}=\sum_{k l}\left[\frac{\partial \xi(\pi)}{\partial \pi_{i}}\right]_{k}\left[\boldsymbol{C}^{-1}\right]_{k l}\left[\frac{\partial \xi(\pi)}{\partial \pi_{j}}\right]_{l}$,

which is exact in the case of pure Gaussian statistics, but may be used as a good approximation for the valley in parameter space in which the minimum of $-\log \mathcal{L}$ lies. Again, $\boldsymbol{C}$ is the covariance of the measured data points and $\xi(\pi)$ the vector of modelled data points in absence of noise.

\subsubsection{Application of the Fisher formalism}

Now we use the Fisher formalism to estimate constraints on various combinations of parameters, with different degrees of redshift binning. First, we evaluate Eq. (38) using the covariance matrix from our fiducial survey consisting of 10 independent sub-fields, $N_{z}=2$ (with $z_{\text {cut }}=1.25$ ), and $N_{\Delta \theta}=65$. The procedure is repeated for the covariance matrix for the coarser $N_{z}=1$ binning. Table 2 shows the percentage error for $N_{z}=2$ as opposed to $N_{z}=1$ for the same set of free and fixed parameters.

We extend the treatment to a larger number of redshift bins, in the context of the survey consisting of 1 sub-field. Again Eq. (38) is calculated, this time using the covariance matrices from the simulations with $N_{z}=4$, and those from coarser binning $\left(N_{z}=3,2,1\right)$ of this data set. Table 3 lists the errors for $N_{z}=4,3,2$ as a percentage of the $N_{z}=1$ error.

In order to investigate the degeneracies of the parameter estimates, we concentrate on the case that no priors are given. For this particular situation, the gain by introducing redshift binning is largest (see Tables 2 and 3). In Fig. 8 we plot the correlations of the errors in the parameter estimates for different pairs of parameters and different numbers of redshift bins. If more than one redshift binning for the same number of redshift bins is available in our data set, we indicate the scatter of correlation coefficients by error bars. Some scatter indicates that the correlations can be changed slightly by varying the bin limits. The strong correlation between the estimates of $\Omega_{\mathrm{m}}$ and $\sigma_{8}$ is only marginally affected by redshift binning. This is also the case for fixed $\Gamma$ and/or $\Omega_{\Lambda}$ (not shown).

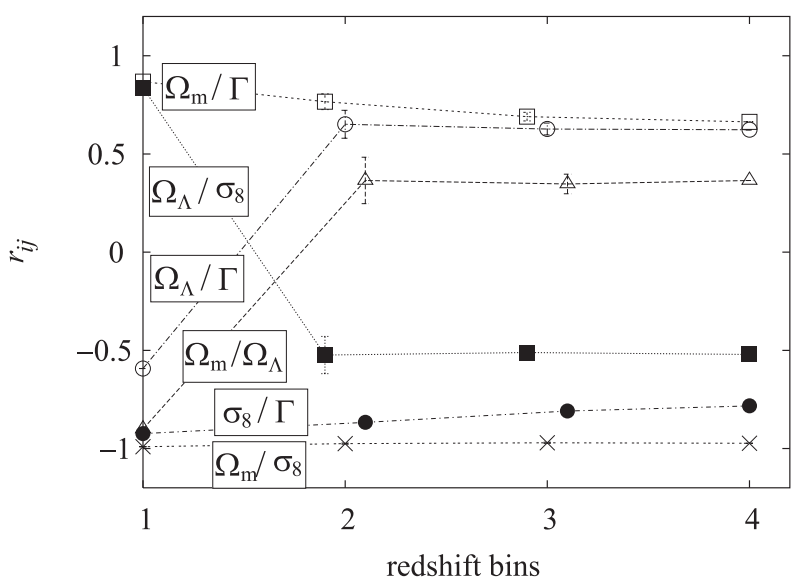

Fig. 8. Correlations of the errors in the parameter estimates for different pairs of parameters and numbers of redshift bins as derived from the Fisher matrix; only the case with no fixed priors is considered. Error bars denote the variance in the correlations for the different redshift binnings for the same number of bins (only for 2 and 3 bins). The data points are slightly shifted to avoid overlapping.

\section{Discussion}

The average shear correlation functions obtained from our numerical simulations are in good agreement with those obtained analytically, as was illustrated in Fig. 4. We also pointed out that their covariance is compatible with Schneider et al. (2002a) and Kilbinger \& Schneider (2003).

Our treatment is only strictly valid for Gaussian density fields and is a good approximation for scales greater than $\sim 10^{\prime}$, giving a lower limit on the covariance at smaller scales (e.g. van Waerbeke et al. 2002). A more accurate covariance matrix is possible, though. According to Schneider et al. (2002a) (Sect. 4 therein), the covariance matrix of $\xi_{+}$may be decomposed into three terms

$C_{i j}=\sigma_{\epsilon}^{2} X_{i j}+\sigma_{\epsilon}^{4} Y_{i j}+Z_{i j}$,

where $X, Y$ and $Z$ are some functions. $X$ and $Y$ are functions of the two-point correlation of cosmic shear and consequently insensitive to non-Gaussian features of the field. $Z$, however, depends linearly on the four-point correlation of cosmic shear which in Schneider et al. (2002a) is worked out by assuming a Gaussian field; this factorises $Z$ into a sum of products of twopoint correlators only. In the hierarchical clustering regime, the four-point correlation of the random field differs from that value only by a constant scale-independent factor $Q$, the socalled hierarchical amplitude (see e.g. Bernardeau et al. 2002). Thus, hierarchical clustering increases the component $Z$ simply by the factor $Q$. This could be included in $C_{i j}$ by the following two steps: i) calculating $C_{i j} \equiv C_{i j}^{(1)}$ by setting the intrinsic noise $\sigma_{\epsilon}=0$, ii) recalculating $C_{i j} \equiv C_{i j}^{(2)}$ this time with the intrinsic noise turned on. The final covariance matrix $C_{i j}$, accounting for $Q$, is obtained by

$C_{i j}=C_{i j}^{(1)}(Q-1)+C_{i j}^{(2)}$.

As an illustration of the use of numerically derived covariance matrices, we have considered to what degree redshift 
Table 2. Uncertainties in the parameter estimates according to the Fisher formalism, for our fiducial survey of 10 uncorrelated subfields. The first data set (2binsIV) with $N_{z}=2$ and $z_{\text {cut }}=1.25$ is used. Columns with dots "." denote fixed parameters (strong priors). Uncertainties in the top panel are absolute values for a single redshift bin. Those in the lower panel are for $N_{z}=2$, quoted as a percentage of the single redshift bin $\left(N_{z}=1\right)$ case. For instance, with no fixed parameters, $\Delta \Omega_{\Lambda}=0.26$ with $N_{z}=2$ (i.e. $13 \%$ of the $N_{z}=1$ error). $\left|\boldsymbol{F}^{-1}\right|$ denotes the determinant of the inverse of the Fisher matrix; its square root is proportional to the volume of the error ellipsoid in parameter space. The $n$th root, with $n$ being the number of free parameters, defines a typical size of the error ellipsoid; this size is proportional to the geometric mean of the lengths of the principal ellipsoid axes.

\begin{tabular}{ccccc}
\hline \hline$\Delta \Omega_{m}$ & $\Delta \Omega_{\Lambda}$ & $\Delta \sigma_{8}$ & $\Delta \Gamma$ & $\sqrt{\left|\boldsymbol{F}^{-1}\right|^{1 / n}}$ \\
\hline 0.9 & 2.0 & 1.2 & 0.4 & 0.16 \\
$\cdot$ & 0.5 & 0.1 & 0.1 & 0.09 \\
0.2 &. & 0.4 & 0.2 & 0.04 \\
0.09 & 0.7 &. & 0.08 & 0.08 \\
0.3 & 1.0 & 0.3 &. & 0.13 \\
& & & & \\
0.06 & 0.5 &. &. & 0.09 \\
0.08 &. & 0.1 &. & 0.05 \\
0.02 & $\cdot$ & $\cdot$ & 0.07 & 0.03 \\
$\cdot$ & 0.3 & 0.06 &. & 0.09 \\
$\cdot$ & 0.1 &. & 0.06 & 0.08 \\
. &. & 0.03 & 0.07 & 0.04
\end{tabular}

\begin{tabular}{|c|c|c|c|c|}
\hline 0.02 & . & . & . & 0.15 \\
\hline . & 0.10 & . & . & 0.10 \\
\hline . & . & 0.02 & . & 0.02 \\
\hline . & . & . & 0.06 & 0.06 \\
\hline $13 \%$ & $13 \%$ & $17 \%$ & $26 \%$ & $47 \%$ \\
\hline . & $52 \%$ & $57 \%$ & $64 \%$ & $72 \%$ \\
\hline $53 \%$ & $\cdot$ & $52 \%$ & $49 \%$ & $73 \%$ \\
\hline $43 \%$ & $38 \%$ & . & $68 \%$ & $65 \%$ \\
\hline $32 \%$ & $24 \%$ & $46 \%$ & . & $57 \%$ \\
\hline $50 \%$ & $45 \%$ & . & . & $64 \%$ \\
\hline $86 \%$ & . & $85 \%$ & . & $88 \%$ \\
\hline $89 \%$ & . & . & $81 \%$ & $85 \%$ \\
\hline . & $65 \%$ & $71 \%$ & . & $76 \%$ \\
\hline . & $80 \%$ & . & $81 \%$ & $81 \%$ \\
\hline . & . & $87 \%$ & $80 \%$ & $84 \%$ \\
\hline $85 \%$ & . & 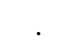 & . & $85 \%$ \\
\hline . & $81 \%$ & . & . & $81 \%$ \\
\hline$\cdot$ & . & $89 \%$ & . & $89 \%$ \\
\hline - & $\cdot$ & . & $82 \%$ & $82 \%$ \\
\hline
\end{tabular}

information tightens the confidence regions on cosmological parameters. Note that we required rather crude redshift information: photometric redshift estimates for sources can be
Table 3. The notation is identical to Table 2 . The second data set was used to obtain these values, with various binnings in redshift denoted by the final column, and with covariance matrices calculated for 1 subfield. Uncertainties are again quoted as a percentage of that for $N_{z}=1$.

\begin{tabular}{|c|c|c|c|c|c|}
\hline$\Delta \Omega_{m}$ & $\Delta \Omega_{\Lambda}$ & $\Delta \sigma_{8}$ & $\Delta \Gamma$ & $\sqrt{\left|\boldsymbol{F}^{-1}\right|^{1 / n}}$ & data se \\
\hline $16 \%$ & $14 \%$ & $19 \%$ & $26 \%$ & $41 \%$ & 2bins \\
\hline $17 \%$ & $14 \%$ & $21 \%$ & $32 \%$ & $42 \%$ & 2 binsI \\
\hline $21 \%$ & $18 \%$ & $30 \%$ & $53 \%$ & $49 \%$ & 2 binsII \\
\hline $85 \%$ & . & $84 \%$ & . & $88 \%$ & 2bins \\
\hline $80 \%$ & . & $80 \%$ & . & $85 \%$ & 2binsI \\
\hline $88 \%$ & . & $89 \%$ & . & $91 \%$ & 2 binsII \\
\hline $74 \%$ & . & . & $70 \%$ & $79 \%$ & 2bins \\
\hline $75 \%$ & . & . & $74 \%$ & $82 \%$ & 2 binsI \\
\hline \multirow[t]{4}{*}{$92 \%$} & . & . & $91 \%$ & $92 \%$ & 2binsII \\
\hline & . & $67 \%$ & $63 \%$ & $76 \%$ & 2bins \\
\hline & . & $71 \%$ & $69 \%$ & $79 \%$ & 2 binsI \\
\hline & . & $90 \%$ & $87 \%$ & $91 \%$ & 2 binsII \\
\hline $13 \%$ & $11 \%$ & $16 \%$ & $23 \%$ & $34 \%$ & 3bins \\
\hline $13 \%$ & $12 \%$ & $16 \%$ & $22 \%$ & $36 \%$ & 3binsI \\
\hline $14 \%$ & $11 \%$ & $18 \%$ & $27 \%$ & $36 \%$ & 3binsII \\
\hline $73 \%$ & . & $75 \%$ & . & $79 \%$ & 3bins \\
\hline $73 \%$ & . & $73 \%$ & . & $79 \%$ & 3binsI \\
\hline $72 \%$ & . & $73 \%$ & . & $78 \%$ & 3binsII \\
\hline $68 \%$ & . & . & $65 \%$ & $73 \%$ & 3bins \\
\hline $67 \%$ & . & . & $64 \%$ & $74 \%$ & 3binsI \\
\hline \multirow[t]{4}{*}{$69 \%$} & . & $\cdot$ & $67 \%$ & $74 \%$ & 3binsII \\
\hline & . & $62 \%$ & $58 \%$ & $70 \%$ & 3bins \\
\hline & . & $61 \%$ & $57 \%$ & $70 \%$ & 3binsI \\
\hline & . & $65 \%$ & $61 \%$ & $72 \%$ & 3binsII \\
\hline $11 \%$ & $9 \%$ & $14 \%$ & $19 \%$ & $31 \%$ & 4bins \\
\hline $65 \%$ & . & $67 \%$ & . & $71 \%$ & 4bins \\
\hline \multirow[t]{2}{*}{$61 \%$} & . & . & $57 \%$ & $66 \%$ & 4bins \\
\hline & . & $55 \%$ & $51 \%$ & $63 \%$ & 4bins \\
\hline
\end{tabular}

obtained from multi-colour observations, with typical accuracies in $\sigma_{z}$ of $\sim 0.1$ or better (e.g. Bolzonella et al. 2000).

We have investigated the information contained in $\xi_{+}$, rather than the shear (or equivalently convergence) power spectrum $P_{K}$, since it is directly obtained from the statistics of the distorted images of distant galaxies. Various estimators of $P_{K}$ have been proposed, requiring the spatial distribution of the shear (e.g. Kaiser 1998; Hu \& White 2001) or the shear correlation functions (Schneider et al. 2002a). Note that a calculation similar to ours but using $\hat{P}_{\kappa}$ would formally require one 
to use the full covariance matrix for the associated estimator. However, as noted in Schneider et al. (2002a), the band-power estimates for $\hat{P}_{\kappa}$ do decorrelate rather quickly.

The first data set consists of 2 redshift bins, and covariance matrices estimated with and without binning for a survey with 10 uncorrelated $\left(1.25^{\circ}\right.$ on a side) sub-fields (i.e. selected from different realisations). Constraints on pairs of cosmological parameters using a likelihood treatment, with and without redshift information, are shown in Figs. 5-7. Since our goal here is to study the benefit of redshift information in cosmic shear studies, we do not adopt priors from WMAP or other probes of large scale structure which might confuse the issue. In both cases the redshift distribution is assumed to be known. Assigning sources to 2 redshift bins tightens the confidence regions in all cases.

Noteworthy are the tightened upper limits on $\Gamma$ in the $\Gamma-\sigma_{8}$ and $\Omega_{\mathrm{m}}-\Gamma$ planes when binning is employed. The constraints on $\Gamma$ in both planes are rather asymmetric, with the confidence regions being more extended towards high $\Gamma$ values. $\Gamma$ determines the location of the peak in the matter power spectrum, and having extra redshift information places tighter constraints on this - there is a degeneracy between $\Gamma$ and the mean source redshift $\langle z\rangle$ such that a larger $\Gamma$ would be compensated for by a smaller $\langle z\rangle$. Recall that $\Gamma$ is not a fundamental quantity; in the limit of zero baryons, $\Gamma=\Omega_{\mathrm{m}} h$, which is modified to $\Gamma=\Omega_{\mathrm{m}} h \exp \left(-\Omega_{\mathrm{b}}\left(1+\sqrt{2 h} / \Omega_{\mathrm{m}}\right)\right)$, if $\Omega_{\mathrm{b}}$, the present baryon density, is accounted for in the transfer function (Sugiyama 1995). With our strong priors (hence fairly tight constraint on $\Omega_{\mathrm{m}}$ or $\sigma_{8}$ ), the error in $\Gamma$ roughly translates into an error in $h$, so redshift binning decreases the upper limit on $h$.

In the $\Omega_{\mathrm{m}}-\sigma_{8}$ plane we obtain the familiar "banana" shaped confidence regions, tightened with the inclusion of redshift binning. It is difficult to directly compare our constraints to real surveys with different observational conditions; however, our confidence regions are roughly compatible with those of van Waerbeke et al. (2001) allowing for these differences.

To explore a wider range of parameter combinations, we employed the Fisher formalism since this allows one to easily obtain error estimates and investigate degeneracies. We used the covariance matrices estimated from the first data set, again for 10 sub-fields as described above. Table 2 shows to what extent the errors on various parameters are improved for $N_{z}=2$ compared with $N_{z}=1$. Note that these results depend on the cosmological model and power spectrum of our fiducial model. Redshift binning is particularly helpful when fewer strong priors are assumed, compared with the case when only one or two of the parameters are allowed to vary. In the case where $\Omega_{\mathrm{m}}$, $\Omega_{\Lambda}, \sigma_{8}$ and $\Gamma$ are free, errors are a factor of roughly 4 to 8 smaller when $N_{z}=2$. As we adopt more strong priors, redshift binning becomes progressively less beneficial. For example, if we consider parameter combinations where either $\Omega_{\mathrm{m}}$ or $\sigma_{8}$ are assumed to be perfectly known, this breaks a strong degeneracy otherwise present; the decrease in errors when $N_{z}=2$ are therefore not so great as one might have anticipated. Another interesting trend is that the constraint of $\Omega_{\Lambda}$ seems to be most favourably affected by redshift binning, perhaps because it is important to the growth rate of structure at redshifts $z \sim 1$.
How does Fisher analysis compare with the likelihood treatment? Fisher analysis should be seen as a way to estimate errors and investigate degeneracies, but does not propose to reveal the detailed behaviour of confidence regions far from the fiducial model - for instance the asymmetric constraints in the $\Gamma-\Omega_{\mathrm{m}}$ or $-\sigma_{8}$ planes, or the "banana" shaped constraints in the $\Omega_{\mathrm{m}}-\sigma_{8}$ plane. Nevertheless, the differences in the constraints on the parameter pairs drawn from $\Omega_{\mathrm{m}}, \sigma_{8}$ and $\Gamma$ are roughly consistent with the full likelihood treatment discussed above.

Using the second data set consisting of 4 redshift bins, for a survey consisting of 1 sub-field, we obtained covariance matrices corresponding to various redshift cuts for 2 and 3 redshift bins. Parameter constraints are again tightened going from $N_{z}=1$ to $N_{z}=4$. Considering again for example the case where all parameters are free, the most dramatic change is already seen going from $N_{z}=1$ to $N_{z}=2$. In the case of $N_{z}=2$, with the lowest redshift cut $z_{\text {cut }}=0.75$ (2binsIII), parameter constraints are weakest. When higher redshift sources are isolated $\left(z_{\text {cut }}=1.5,2.25\right)$ the constraints are similar, and which is the better choice depends on the combination of parameters considered. For 3 redshift bins, the combinations of cuts in redshift lead to very similar error estimates.

At some point further sub-division into redshift bins does not lead to improved constraints on parameters (Hu 1999). This limit must be determined for the survey and cosmological parameters in question. For simplicity consider the case of $N_{z}=2: \xi_{\mathrm{L}}$ and $\xi_{\mathrm{U}}$ are correlated since sources in the upper redshift bin are also sensitive to lensing by structure at $z<z_{\text {cut }}$. A measure of this correlation is provided by a correlation parameter, $p=\xi_{\mathrm{LU}} /\left(\xi_{\mathrm{L}} \xi_{\mathrm{U}}\right)^{0.5}$, also used by Hu (1999) in the context of power spectra. $p=1$ and $p=0$ indicate complete correlation and lack of correlation respectively. For our simulations, $0.83<p<0.93$ for the range over which the correlation functions are calculated, with $\bar{p}=0.87$ taking the mean over all $\Delta \theta$ bins.

The improvement of the parameter constraints is clearly also a function of how the redshift intervals are set. This can already be seen in Table 3 where we calculated different binnings with two and three redshift bins. We expect that there is an optimal way to bin the data. The intention of this paper, however, is to present a fast method for calculating the covariance matrix of the cosmic shear correlation estimator. This issue will be explored in a forthcoming publication. In practise, this question probably does not arise anyway, because there one would take as many redshift bins as possible, their number being determined by the accuracy of the redshift estimator.

As described above, one of our fiducial surveys is composed of 10 uncorrelated sub-fields. Another possibility is that a single contiguous patch of sky is targeted primarily for another science goal; such a survey might consist of 10 sub-fields drawn from the same large field. The sub-fields might in that case be selected so as to avoid bright stars, chip boundaries or defects. These sub-fields would be correlated to some extent, meaning that taking $m$ rather than $n(<m)$ sub-fields does not decrease the covariance by a factor of $m / n$, which would be the case if they were independent. The degree to which the sub-fields are correlated is accounted for directly in the covariance matrix. However, it is instructive to have an estimate of 
this, using the (ensemble average) covariance matrix for a survey composed of 10 correlated as opposed to 10 uncorrelated sub-fields. The ratio of the diagonal elements of the correlated and uncorrelated geometry covariance matrices ranges between $\approx 1.1-1.4$, from the lower to upper redshift bin. Of course, the amount of correlation between sub-fields also depends on their geometry, so this has to be estimated for the survey in question.

\section{Conclusions}

In preparation for upcoming cosmic shear surveys, the main purpose of this paper was to demonstrate that it is possible to rapidly simulate surveys using a Monte Carlo method. This enables one to obtain accurate full covariance matrices for the shear two-point correlation function $\xi_{+}$, estimated from arbitrary survey geometries and with the sources binned in redshift. Averaging over many independent realisations enables us to take into account cosmic variance. As a first application, we estimated the extent to which redshift information for sources used in a cosmic shear analysis improves constraints on parameters derived from the estimated shear two-point correlation function $\hat{\xi}_{+}$.

A likelihood analysis in the $\Omega_{\mathrm{m}}-\sigma_{8}, \Omega_{\mathrm{m}}-\Gamma$ and $\Gamma-\sigma_{8}$ planes shows that separating the sources into two redshift bins enables tighter constraints to be placed on parameters. Considering a wider range of parameter combinations in the context of a Fisher analysis reveals that redshift information is particularly advantageous in cases where few strong priors are assumed. When $\Omega_{\mathrm{m}}, \sigma_{8}, \Gamma$ and $\Omega_{\Lambda}$ are free parameters, having 2 (4) redshift bins tightens errors on parameters by a factor of 4-8 ( 5-10). Most improvement on error estimates occurs going from $N_{z}=1$ to $N_{z}=2$. In general, for the combinations of free and fixed parameters explored, $\Omega_{\Lambda}$ seems to benefit most from redshift binning,

One might ask why cosmic shear is of interest, in the light of the recent WMAP results (e.g. Bennett et al. 2003), which suggest that the era of precision cosmology is already upon us; there are several facets to consider. Cosmic shear has the power to break degeneracies inherent to $\mathrm{CMB}$ data (e.g. $\mathrm{Hu}$ $\&$ Tegmark 1999), for instance the angular diameter distance degeneracy (e.g. Efstathiou \& Bond 1999). It also provides a completely independent cross-check of cosmological parameters, based on equally well understood but different physical principles. Besides this, as is the case with the CMB, the interpretation of results requires no assumption about the bias between luminous tracers and the underlying dark matter distribution which plagues, for example, galaxy redshift surveys. In addition, with redshift estimates for sources in upcoming large cosmic shear surveys, lensing has the potential to see beyond the radially projected convergence power spectrum, becoming sensitive to the evolution and growth of structure in the Universe.

Acknowledgements. We would like to thank Martin Kilbinger, Ludo van Waerbeke and Wayne $\mathrm{Hu}$ for helpful discussions. This work was supported by the Deutsche Forschungsgemeinschaft under the Graduiertenkolleg 787 and the project SCHN 342/3-1, and by the German Ministry for Science and Education (BMBF) through the DLR under the project 50 OR 0106.

\section{Appendix A: Switching from a finer to a coarser redshift binning}

Here we show how the auto- and cross-correlations of the cosmic shear from a finer redshift binning are related to the autoand cross-correlations obtained from a coarser redshift binning (by combining the finer bins). The relations of this section ensure that we only have to make simulated data of the finest redshift binning, since the corresponding data with less redshift information can always be related to the 3D-correlations of the cosmic shear of this case.

In a first step, we turn to the auto-correlation $\xi_{ \pm}$of a new redshift bin, neglecting for a moment the cross-correlations to the other new redshift bins. $\xi_{ \pm}$is according to the Eqs. (1) and (7) a function that linearly depends on $\bar{W}^{2}(w)$

$$
\begin{aligned}
\xi_{ \pm}(\theta)= & \frac{9 H_{0}^{4} \Omega_{m}^{2}}{4 c^{4}} \\
& \times \int \frac{\mathrm{d} \ell \ell}{2 \pi} \int_{0}^{w_{h}} \mathrm{~d} w \frac{\bar{W}^{2}(w)}{a^{2}(w)} J_{0,4}(\ell \theta) P_{\delta}\left(\frac{\ell}{f(w)}, w\right) .
\end{aligned}
$$

If we split the redshift distribution $p(w)$ of the source galaxies into disjunct parts, like

$$
\begin{aligned}
p(w) & =\sum_{i} q^{(i)}(w) \\
q^{(i)}(w) & \equiv \begin{cases}p(w) & \text { for } w \in\left[w_{i-1}, w_{i}\right] \\
0 & \text { else }\end{cases}
\end{aligned}
$$

with $w_{i}\left(z_{i}\right)$ in the sense of Fig. 1, we expand with the notation of Eqs. (4) the function $\bar{W}$ in the following manner

$$
\begin{aligned}
\bar{W}^{2}(w) & =\sum_{i j} n_{i} n_{j} \bar{W}^{(i)}(w) \bar{W}^{(j)}(w) \\
& =\sum_{i} n_{i}^{2}\left[\bar{W}^{(i)}(w)\right]^{2}+2 \sum_{i>j} n_{i} n_{j} \bar{W}^{(i)}(w) \bar{W}^{(j)}(w) .
\end{aligned}
$$

As the $q^{(i)}$ defined here are not normalised, but the corresponding redshift distributions $p^{(i)}$ in the definition for $\bar{W}^{(i)}(w)$ are, we introduce the normalisation factors

$n_{i} \equiv \int_{w_{i-1}}^{w_{i}} \mathrm{~d} w q^{(i)}(w)$

telling us what fraction of the distribution inside the new bin is contained in its subdivisions $q^{(i)}$. Translating Eq. (A.3) to the power spectra gives, leaving out the arguments in $\ell$

$P_{\kappa}=\sum_{i} n_{i}^{2} P_{\kappa}^{(i i)}+2 \sum_{i>j} n_{i} n_{j} P_{\kappa}^{(i j)}$

Similarly, we get for the cosmic shear auto-correlation $\tilde{\xi}$ (actually for all linear functions of $P_{\kappa}^{(i j)}$; hence the dropped index “ $\pm ”)$ :

$\tilde{\xi}=\sum_{i} n_{i}^{2} \xi_{i i}+2 \sum_{i>j} n_{i} n_{j} \xi_{i j}$

where $\xi_{i i}$ are the auto-correlations for the sub-bins, $\xi_{i j}$ are the cross-correlations of the cosmic shear between the sub-bins. This equation tells us, therefore, how we have to combine the 
cosmic shear correlations of the sub-bins when we are switching from a finer to a coarser redshift binning of the data.

What about the cross-correlations between the new redshift bins if we decide to switch to a binning with more than one redshift bin? This case is treated like the foregoing one, except that it is slightly more general. Assume we focus on two new redshift bins $k$ and $l$ consisting of data from a finer redshift binning:

$p_{k}(w)=\sum_{i} q_{k}^{(i)}(w) ; \quad p_{l}(w)=\sum_{j} q_{l}^{(j)}(w)$,

where $p_{k}$ is the redshift distribution inside the new bin $k$ and $p_{l}$ the same for the new bin $l$. Bin $k$ combines $q_{k}^{(i)}$ and the bin $l$ combines $q_{l}^{(j)}$ from a finer binning, respectively. Using the same arguments as before, we obtain the following relation between the cosmic shear cross-correlation between the new redshift bins $\tilde{\xi}_{k l}$, and the cross-correlations $\xi_{i j}^{(k l)}$ between their components:

$\tilde{\xi}_{k l}=\sum_{i j} n_{i}^{(k)} n_{j}^{(l)} \xi_{i j}^{(k l)}$

$n_{i}^{(k)}$ and $n_{j}^{(l)}$ are the normalisations for the sub-bins. This equation is, of course, the generalisation of Eq. (A.6).

\section{References}

Bacon, D. J., Refregier, A. R., \& Ellis, R. S. 2000, MNRAS, 318, 625 Bardeen, J. M., Bond, J. R., Kaiser, N., \& Szalay, A. S. 1986, ApJ, 304, 15

Bartelmann, M., \& Schneider, P. 2001, Phys. Rep., 340, 291

Bennett, C., et al. 2003, ApJS, 148, 1

Bernardeau, F., van Waerbeke, L., \& Mellier, Y. 1997, A\&A, 322, 1

Bernardeau, F., Colombi, S., Gaztańaga, E., \& Scoccimarro, R. 2002, $\mathrm{PhR}, 367,1$

Blandford, R. D., Saust, A. B., Brainerd, T. G., \& Villumsen, J. V. 1991, MNRAS, 251, 600

Bolzonella, M., Miralles, J.-M., \& Pell/o, R. 2000, A\&A, 363, 476
Efstathiou, G., \& Bond, J. R. 1999, MNRAS, 304, 75

Fisher, R. A. 1935, J. Roy. Stat. Soc., 98, 39

Hamilton, A. J. S., Matthews, A., Kumar, P., \& Lu, E. 1991, ApJ, 374,1

Higham, N. J. 1997, Numerical Algorithms, 15, 227

Hu, W., \& Tegmark, M. 1999, ApJ, 514, L65

Hu, W. 1999, ApJ, 522, L21

Hu, W., \& White, M. 2001, ApJ, 554, 67

Huterer, D. 2002, Phys. Rev. D, 65, 063001

Jain, B., Seljak, U., \& White, S. D. M. 2000, ApJ, 530, 547

Kaiser, N. 1992, ApJ, 388, 272

Kaiser, N. 1998, ApJ, 498, 26

Kaiser, N., Wilson, G., \& Luppino, G. 2000 [astro-ph/0003338]

Kilbinger, M., \& Schneider, P. 2004, A\&A, 413, 465

King, L. J., \& Schneider, P. 2003, A\&A, 398, 23

Miralda-Escudé, J. 1991, ApJ, 380, 1

Peacock, J. A., \& Dodds, S. J. 1996, MNRAS, 280, L19

Peacock, J. A. 2001, Cosmological Physics (Cambridge University Press), 503

Pen, U. L., \& Zhang, L. L. 2003 [astro-ph/0305447]

Press, W. H., Tokolsky, S. A., Vetterling, W. T., \& Flannery, B. P. 1992, Numerical Recipes (Cambridge University Press)

Schneider, P., van Waerbeke, L., Jain, B., \& Kruse, G. 1998, MNRAS, 296, 873

Schneider, P., van Waerbeke, L., Kilbinger, M., \& Mellier, Y. 2002a, A\&A, 396, 1

Schneider, P., van Waerbeke, L., \& Mellier, Y. 2002b, A\&A, 389, 729

Smail, I., Hogg, D., Yan, L., \& Cohen, J. 1995, ApJ, 449, 105L

Sugiyama, N. 1995, ApJS, 100, 281

Tegmark, M., Taylor, A. N., \& Heavens, A. F. 1997, ApJ, 480, 22

van Waerbeke, L., Mellier, Y., Erben, T., et al. 2000, A\&A, 358, 30

van Waerbeke, L., Mellier, Y., Radovich, M., et al. 2001, A\&A, 374, 757

van Waerbeke, L., Mellier, Y., Pell/o, R., et al. 2002, A\&A, 393, 369

van Waerbeke, L., \& Mellier, Y. 2003, Lecture given at Aussois Winter School, 1/2003 [astro-ph/0305089]

Wambsganss, J., Cen, R., \& Ostriker, J. P. 1998, ApJ, 494, 29

White, M., \& Hu, W. 2000, ApJ, 537, 1

Wittman, D. M., Tyson, J. A., Kirkman, D., Dell'Antonio, I., \& Bernstein, G. 2000, Nature, 405, 143 